\title{
Averaged cortical potentials (lambda responses) time-locked to onset and offset of saccades
}

\author{
AKIHIRO YAGI \\ Industrial Products Research Institute, Tsukuba Science City, Ibaraki, Japan
}

\begin{abstract}
The purpose of the experiment was to examine the assumption that the lambda response would be associated with offset of the saccade. The occipital EEG and eye movements were recorded under the condition in which the subjects looked at the stimulus object freely. EEGs triggered at onset and offset of saccades were averaged separately by a computer. Lambda responses appeared to be more definite and were larger in amplitude in onset averages than in offset averages. The result showed that the lambda response was associated with offset of the saccade, and not with its onset. It is suggested that the lambda response might be a kind of visual evoked potential elicited by the afferent inflow beginning at fixation pause.
\end{abstract}

When occipital EEGs were averaged on the basis of the moment of the saccade, complex potentials (the lambda complex) consisting of some components can be obtained. The most prominent component following the saccade is referred to as the lambda response (Yagi, 1979a). The lambda response is not found when the subject closes his eyes, when he is in the dark, or when he observes a uniform field (Scott, Goethuysen, \& Bickford, 1967). The response has been interpreted as a cortical response to retinal stimulation that results from shifting of the image across the retina (Barlow \& Cigánek, 1969). Recently, we found that the peak latency of the lambda response measured from onset of the saccade increased in proportion to the saccade size (Yagi, 1979a, 1979b). These results showed that the lambda response was associated with offset of the saccade, but not with its onset (Yagi \& Ohtani, 1977). The moment of offset of the saccade is the beginning of the next fixation pause. Therefore, results mentioned above suggest that the lambda response is associated with onset of the fixation pause.

The duration of the saccade is not constant when the subject observes the stimulus object freely (Yarbus, 1967). Therefore, it would be expected that two different types of potentials would be obtained when EEGs are averaged separately at the onset and at the offset of saccades in a free observing situation. As Brazier (1964) pointed out in her explanation of averaged evoked potentials, waves perfectly aligned produce the largest possible average while variability reduces amplitude. Components associated with onset of saccades will appear to be more definite and

This research was supported by a Special Research Projects grant from the Agency of Industrial Science and Technology, and a grant in aid for Science Research from the Ministry of Education in Japan. Requests for reprints should be sent to Akihiro Yagi, Industrial Products Research Institute, 1-1-4, Yatabemachi-Higashi, Tsukuba Science City, Ibaraki, 305, Japan. larger in amplitude in onset averages than in offset averages.

The purpose of the present experiment was to compare the differences in potential components obtained by averaging EEGs time-locked to onset and offset of saccades in a free observing situation and to confirm the assumption that the lambda response would be associated with offset of the saccade.

\section{METHODS}

\section{Subject}

The subjects were 12 normal human adults, aged 21 to 29 years, who had binocular visual acuities of better than 1.0. One of them, however, was excluded from the analysis of data because of frequent eye blinking.

\section{Procedure}

After electrode placement, the subject was seated in front of a Goldman perimeter in a soundproof and shielded room, with his chin and forehead in contact with a headrest to limit movement. The distance between the surface of the perimeter and the subject's nasion was $30 \mathrm{~cm}$. Inside the perimeter, small black squares, with sides of $1 \mathrm{deg}$, were placed at random on the white ground. The subject was instructed to look at the random pattern and to change his eye position freely. The luminance level of the white part was $4.0 \mathrm{~mL}$, and of the black, $.40 \mathrm{~mL}$. The trial lasted about $4 \mathrm{~min}$.

\section{Recording}

EEG was recorded from $\mathrm{Oz}$ referred to linked ears. The ground lead was attached to the midline forehead. The signal was amplified with a Nihonkoden amplifier (RM-5) at a low-frequency time constant of $.3 \mathrm{sec}$ and a high-frequency cutoff at $100 \mathrm{~Hz}$. Eye movements were recorded by means of electrooculography (EOG). A pair of $\mathrm{Ag}-\mathrm{AgCl}$ electrodes (Beckman) were placed at the outer canthi of two eyes for horizontal movements. Another pair was placed above and below the right eye for vertical movements and eye blink potentials. The signals were amplified with amplifiers (RM-5) at a low-frequency time constant of $2 \mathrm{sec}$ and a highfrequency cutoff of $300 \mathrm{~Hz}$. EEG and EOGs were recorded on a magnetic tape (Teak, R-280).

\section{Analysis of Data}

EEGs time-locked to onset of saccades. First, EEGs were timelocked to onset of saccades and averaged. The horizontal and the 
vertical EOGs reproduced with tape run forward were differentiated, with the differentiators at a time constant of $.02 \mathrm{sec}$. Both potentiais were fed to input of a vector module (Teledyne Philbrick4352) to obtain the absolute value of the saccade. Whenever the output of the module exceeded a given level $(2 \mathrm{deg})$, a pulse used to trigger a computer (DEC, PDP-11/10) was formed with a monomultivibrator. The positive high spike (more than $200 \mu \mathrm{V}$ ) in the vertical EOG, which was defined as a blink potential, was also transformed into a pulse. The occipital EEG, vector EOGs, and the blink pulse were digitized every $3 \mathrm{msec}$ with separate A-D converters and stored temporarily in buffer memories of the computer. When the eyeblink or the next saccade occurred during sampling EEG, buffer memories were erased. Thus, occipital EEGs from $45 \mathrm{msec}$ before to $255 \mathrm{msec}$ after onset of saccades were obtained without artifacts and averaged 100 times by the computer. After running the average of the five points, the results were recorded on an XY plotter.

EEGs time-locked to offset of saccades. The data were processed in the same manner as described in the previous analysis with tape run backward in order to detect the offsets of saccades. EEGs from $75 \mathrm{msec}$ before to $225 \mathrm{msec}$ after the offset of saccades were averaged 100 times.

\section{RESULTS}

Typical wave forms in the onset (a broken tracing) and offset averages (a solid tracing) are illustrated in Figure 1. The major components, N1, P1, N2, P2, and N3, were obtained in nearly every subject. P1 appeared clearly as a sharp wave in onset averages,

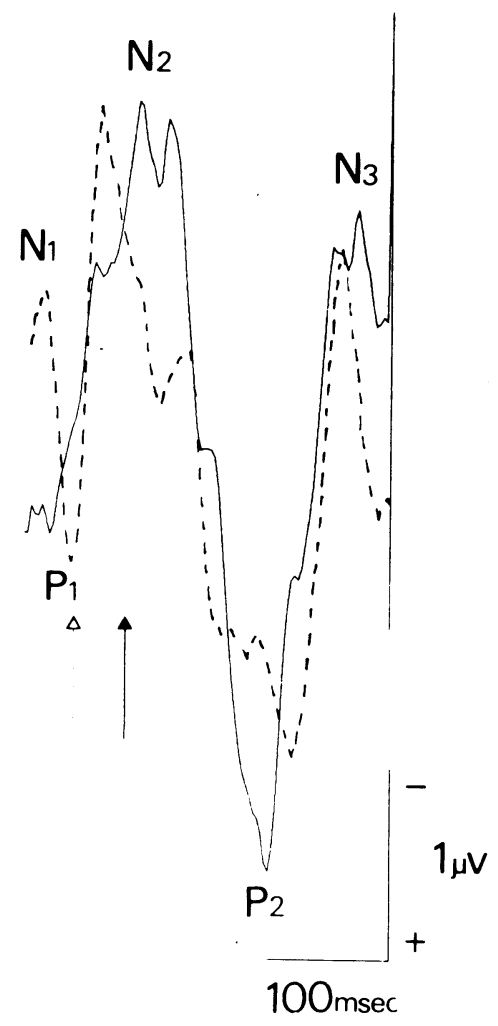

Figure 1. Typical wave forms obtained with onset averages (broken tracing) and with of fset averages (solid tracing). Arrows indicate the moment of saccade onset (broken) and that of saccade offset (solid).

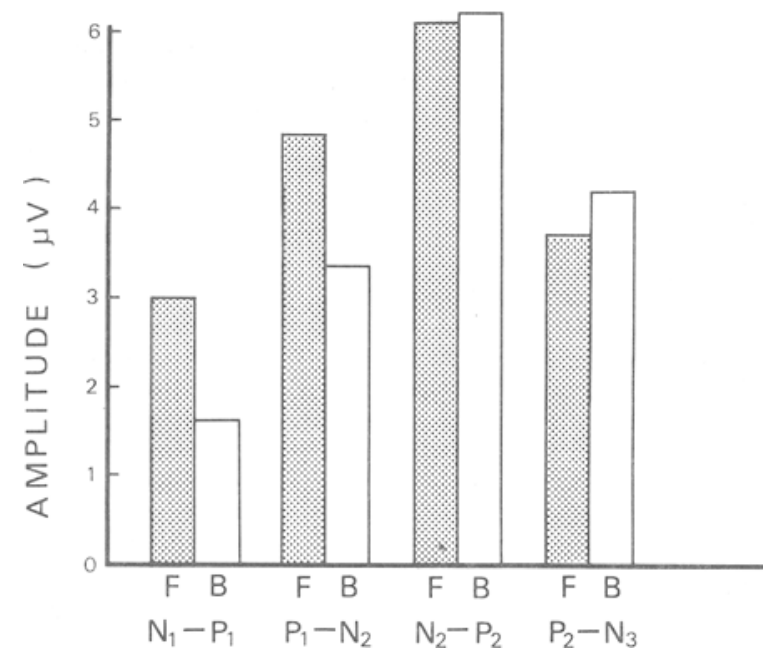

Figure 2. Mean peak-to-peak amplitudes of lambda responses in onset averages (F) and of fset averages (B).

Table 1

Means, Standard Deviations, and Coefficients of Variation of Peak-to-Peak Amplitudes of Lambda Responses in Onset Averages (F) and in Offset Averages (B)

\begin{tabular}{llcc}
\hline & & $\mathrm{F}$ & $\mathrm{B}$ \\
\hline $\mathrm{N} 1-\mathrm{P} 1$ & $\mathrm{M}$ & 2.99 & 1.63 \\
$(\mathrm{n}=9)$ & $\mathrm{SD}$ & \pm 1.29 & \pm 1.16 \\
& $\mathrm{CV}$ & 43.20 & 71.19 \\
$\mathrm{P} 1-\mathrm{N} 2$ & $\mathrm{M}$ & 4.83 & 3.35 \\
$(\mathrm{n}=11)$ & $\mathrm{SD}$ & \pm 1.72 & \pm 1.39 \\
& $\mathrm{CV}$ & 35.7 & 41.6 \\
$\mathrm{~N} 2-\mathrm{P} 2$ & $\mathrm{M}$ & 6.12 & 6.21 \\
$(\mathrm{n}=11)$ & $\mathrm{SD}$ & \pm 1.98 & \pm 1.56 \\
& $\mathrm{CV}$ & 32.3 & 25.2 \\
$\mathrm{P} 2-\mathrm{N} 3$ & $\mathrm{M}$ & 3.72 & 4.21 \\
$(\mathrm{n}=11)$ & $\mathrm{SD}$ & \pm 1.63 & \pm 1.20 \\
& $\mathrm{CV}$ & 43.9 & 28.4 \\
\hline
\end{tabular}

Note-Means and standard deviations given in microvolts.

though it was vague in offset averages. On the other hand, $P 2$ in offset averages was definite, but the peak of P2 was obscured in onset averages. The peak-topeak amplitudes, N2-P2 and P2-N3, were larger in the of fset averages than in the onset averages.

The mean peak-to-peak amplitudes across 11 subjects are shown in Figure 2 and in Table 1. Since 2 of the 11 subjects showed no definite N1 peak, data of only 9 subjects were processed for N1-P1 amplitude.

The mean peak-to-peak amplitudes in the early components (N1-P1 and P1-N2) were significantly larger in their onset averages than in their offset averages $[t(8)=4.616, p<.01$, and $t(10)=4.347, p<.01$, respectively]. On the other hand, the mean peak-topeak amplitudes of the late components (P2-N3) was significantly larger in the offset averages than in the onset averages $[t(10)=2.826, p<.02]$. The N2-P2 
amplitude showed the same tendency, but the difference between the analyses was not significant.

The standard deviation (SD) and the coefficient of variance (CV) of the amplitude are also shown in Table 1. The SD of the P2-N2 amplitude was significantly smaller in its offset averages than in its onset averages $[\mathrm{t}(9)=3.964, \mathrm{p}<.01]$. No significant difference was obtained in the SDs of the other amplitudes. CVs of amplitudes in the early components were smaller in their onset averages than in their offset averages, while CVs of amplitudes in the late components were smaller in their offset averages than in their onset averages. The CV showed the inverse relation to the peak-to-peak amplitude.

The means and the SDs of the saccade duration and the saccade size were $30.2 \mathrm{msec}( \pm 9.26 \mathrm{msec})$ and $4.71 \mathrm{deg}( \pm 1.68 \mathrm{deg})$, respectively.

\section{DISCUSSION}

When occipital EEGs were averaged separately on the basis of onset and offset of saccade, the wave forms obtained with the onset averages were different from those obtained with the offset averages. The most prominent component was obtained with the offset averages and was followed by the saccade. The amplitude of the component, which was identified as the lambda response (Yagi, 1979a), was larger in the offset averages than in the onset averages. As we expected prior to the experiment, these results showed that the lambda response was associated with offset of the saccade and not with its onset. Furthermore, the variability of the lambda response was smaller in amplitude in the offset averages than in the onset averages. The result also indicates that the lambda response is associated with the offset of the saccade.

The moment of offset of the saccade is the beginning of the next fixation pause. In other words, the above results indicate that the lambda response is associated with onset of the fixation pause. When the effective stimulus is presented to the retina, the evoked potential is elicited on the cortex by the afferent inflow from the retina. During the saccade, however, processing of the input information is suppressed by saccadic suppression (Latour, 1962; Volkmann, 1962; Zuber \& Stark, 1966). Therefore, the lambda response might be evoked by the afferent inflow beginning at the fixation pause.

Recently, we have found that the lambda response obtained with offset averages is similar to the visual evoked potential (Yagi, 1977, 1981, Note 1). Wurtz (1969) has found that some of the cortical neurons are excited during the fixation pause and suppressed during the saccade. His finding may represent similar evidence at the neuronal level. These results also support the assumption that the lambda response is a kind of evoked potential that is elicited by afferent inflow during the fixation pause.
Another component with the temporal relation to the saccade was the P1 component whose peak coincided with onset of the saccade. This agrees with results in earlier reports. Alternative explanations of the origin of the component have been proposed. One is that the component would reflect the neural process responsible for the programming and timing of the saccade (Kurtzberg \& Vaughan, 1977), and the other is that the component is of eye-muscle origin (Becker, Hoehne, Iwase, \& Kornhuber, 1972). The finding that the potential started to change prior to onset of the saccade favors the assumption of central origin. A conclusive explanation of the origin of $\mathrm{P} 1$ should be the subject of further study.

\section{REFERENCE NOTE}

1. Yagi, A. Lambda response as an index of visual perception research. Manuscript submitted for publication, 1981.

\section{REFERENCES}

Barlow, J. S., \& CigÁneK, L. Lambda responses in relation to visual evoked responses in man. Electroencephalography and Clinical Neurophysiology, 1969, 26, 183-192.

Becker, W., Hoehne, O., Iwase, K., \& Kornhuber, H. H. Bereitschaftspotential, prämotorische Positivierung und andere Hirnpotentiale bei sakkadischen Augenbewegungen. Vision Research, 1972, 12, 421-436.

Brazier, M. A. B. Evoked responses recorded from the depths of the human brain. Annals of the New York Academy of Sciences, 1964, 112, 33-59.

Kurzberg, D., \& Vaughan, H. G. Electrophysiological observations on the visuomotor system and visual neurosensorium. In J. E. Desmedt (Ed.), Visual evoked potentials in man: New developments. Oxford: Clarendon Press, 1977.

Latour, P. L. Visual threshold during eye movements. Vision Research, 1962, 2, 261-262.

Scott, D. F., Goethuysen, U. G., \& Bickford, R. G. Lambda responses in the human electroencephalogram. Neurology (Minneapolis), 1967, 17, 770-778.

VolkmanN, F. C. Vision during voluntary saccadic eye movements. Journal of the Optical Society of America, 1962, 52, 571-578.

WURTz, R. H. Response of striate cortex neurons to stimuli during rapid eye movements in the monkey. Journal of Neurophysiology, 1969, 32, 975-986.

YAGI, A. Selective attention and lambda responses. Japanese Journal of the Neurosciences Research Association, 1977, 3, 112-113.

YAGI, A. Lambda waves associated with offset of saccadic eye movements: A subject with large lambda waves. Biological Psychology, 1979, 8, 235-238. (a)

YAGI, A. Saccade size and lambda complex in man. Physiological Psychology, 1979, 7, 370-376. (b)

YAGI, A. Visual signal detection and lambda responses. Electroencephalography and Clinical Neurophysiology, 1981, in press.

Yagi, A., \& Ohtani, A. Lambda responses in free eye movements. Japanese Journal of Electroencephalography and Electromyography, 1977, 5, 40.

YARBUS, A. L. Eye movements and vision (B. Haigh, trans.). New York: Plenum, 1967.

ZuBER, B. C., \& SARK, L. Saccadic suppression: Elevation of visual threshold associated with saccadic eye movements. Experimental Neurology, 1966, 16, 65-79.

(Manuscript received March 31, 1981; revision accepted for publication August 18, 1981.) 